\title{
GM-CSF and IL-2 induce specific cellular immunity and provide protection against Epstein-Barr virus lymphoproliferative disorder
}

\author{
Robert A. Baiocchi, ${ }^{1}$ Jacqueline S. Ward, ${ }^{1}$ Lester Carrodeguas, ${ }^{1}$ Charles F. Eisenbeis, ${ }^{1}$ \\ Ruoqi Peng, ${ }^{1}$ Sameek Roychowdhury, ${ }^{1,2}$ Srinivas Vourganti, ${ }^{1}$ Taryn Sekula, ${ }^{1}$ \\ Maggie O’Brien, ${ }^{3}$ Melvin Moeschberger, ${ }^{4}$ and Michael A. Caligiuri ${ }^{1,2,3}$ \\ ${ }^{1}$ Department of Medicine, Division of Hematology/Oncology, \\ ${ }^{2}$ Department of Molecular Virology, Immunology and Medical Genetics, Division of Human Cancer Genetics, \\ ${ }^{3}$ Department of Veterinary Biosciences, and \\ ${ }^{4}$ School of Public Health, Comprehensive Cancer Center, The Ohio State University, Columbus, Ohio, USA
}

Address correspondence to: Michael A. Caligiuri, The Ohio State University, 458A Starling Loving Hall, 320 West 10th Avenue, Columbus, Ohio 43210, USA. Phone: (614) 293-7521; Fax: (614) 293-7522; E-mail: caligiuri-1@ medctr.osu.edu.

Robert A. Baiocchi and Jacqueline S. Ward contributed equally to this work.

Received for publication April 5, 2001, and accepted in revised form July 30, 2001.

\begin{abstract}
Epstein-Barr virus-associated lymphoproliferative disease (EBV-LPD) is a potentially life-threatening complication in immune-deficient patients. We have used the severe combined immune deficient (SCID) mouse engrafted with human leukocytes (hu-PBL-SCID) to evaluate the use of human cytokines in the prevention of EBV-LPD in vivo. Daily low-dose IL-2 therapy can prevent EBV-LPD in the hu-PBL-SCID mouse, but protection is lost if murine natural killer (NK) cells are depleted. Here we demonstrate that combined therapy with human GM-CSF and low-dose IL-2 is capable of preventing EBV-LPD in the hu-PBL-SCID mouse in the absence of murine NK cells. Lymphocyte depletion experiments showed that human NK cells, $\mathrm{CD8}^{+} \mathrm{T}$ cells, and monocytes were each required for the protective effects of GM-CSF and IL-2 combination therapy. This treatment resulted in a marked expansion of human $\mathrm{CD}^{+} \mathrm{CD}^{+}$lymphocytes in vivo. Using HLA tetramers complexed with EBV immunodominant peptides, a subset of these lymphocytes was found to be EBV-specific. These data establish that combined GM-CSF and low-dose IL-2 therapy can prevent the immune deficiencies that lead to fatal EBV-LPD in the hu-PBL-SCID mouse depleted of murine NK cells, and they point to a critical role for several human cellular subsets in mediating this protective effect.
\end{abstract}

J. Clin. Invest. 108:887-894 (2001). DOI: 10.1172/JCI200112932.

\section{Introduction}

Posttransplant lymphoproliferative disease (PTLD) complicates between $2 \%$ and $20 \%$ of solid organ transplants performed annually in the United States. PTLD is universally associated with the Epstein-Barr virus (EBV) and expresses EBV gene products that have been shown to be essential to B lymphocyte transformation and immortalization in vitro and in vivo (1). The incidence of PTLD has been shown to be directly related to a low frequency of EBV-specific cytotoxic T lymphocytes (CTLs) in patients who are iatrogenically immune suppressed after allogeneic bone marrow transplantation (2). Treatment of PTLD consists of reduction in immune suppressive therapy, chemotherapy, surgery, and irradiation. Tumor resolution has been documented after reduction of immune suppressive therapy (3). However, the development of graft versus host disease (GVHD) in bone marrow transplant patients or rejection of the transplanted graft often occurs. There are few data on a single mode of treatment in this disorder; however, there appears to be an overall survival of approximately $30 \%$, with many of these patients losing their organ grafts $(4,5)$. As organ transplants continue to grow in number, and graft survival improves with advances in immune suppressive therapy, PTLD will likely continue to be a significant problem. Although some has been learned from the retrospective analysis of clinical data generated from patients afflicted with PTLD, the utility of an in vivo model to evaluate rational strategies to treat and ultimately prevent EBV-associated PTLD cannot be overstated.

When severe combined immune deficient (SCID) mice are engrafted with peripheral blood leukocytes (hu-PBL-SCID) from healthy human donors who are seropositive for EBV, the majority of hu-PBL-SCID mice spontaneously develop a fatal human $\mathrm{EBV}^{+}$lymphoproliferative disorder (EBV-LPD). These tumors may be monoclonal, oligoclonal, or polyclonal in nature, contain latent and lytic EBV gene products, and have a sur- 
face phenotype and karyotype that most closely resemble B cell tumors of PTLD $(6,7)$. Tumors arising in huPBL-SCID mice are similar to PTLD in that they demonstrate an immunoblastic $B$ cell phenotype, contain EBV DNA, and display a latency type III gene profile. Given the similarities that exist between PTLD in humans and the spontaneous development of human EBV-LPD in the hu-PBL-SCID xenogeneic mouse, this model may provide a unique opportunity to explore the pathogenesis and treatment of PTLD in vivo.

We have used the hu-PBL-SCID mouse to evaluate the prevention and pathogenesis of EBV-LPD in vivo (7-9). We have previously shown that administration of daily low-dose IL-2 can prevent EBV-LPD in the huPBL-SCID mouse and that human $\mathrm{CD}^{+} \mathrm{T}$ cells and murine NK cells are necessary for the IL-2-mediated protective effect (8). Depletion experiments clearly established that mouse NK cells were a critical cellular component of the IL-2-mediated prevention of EBVLPD and that human NK cells could not substitute for this effect in their absence, despite a significant in vivo expansion of this human lymphocyte population. In the current study, we tried to establish protective immunity to prevent EBV-LPD in the hu-PBL-SCID mouse depleted of murine NK cells. We show that the coadministration of human GM-CSF and IL-2 prevents EBV-LPD and that it does so, at least in part, by inducing EBV-specific $\mathrm{CD}^{+}{ }^{+} \mathrm{CD} 8{ }^{+} \mathrm{T}$ cells in vivo.

\section{Methods}

Animals. Six-week-old C.B.17 scid/scid mice were purchased from Taconic Farms (Germantown, Pennsylvania, USA) and housed in a specific pathogen-free environment provided by the Institute Biocontainment Facility. Food supplements and instruments were autoclaved, and all manipulations were performed in a laminar-flow hood. Animals showed no evidence of the leaky phenotype (10), as determined by ELISA assay to evaluate for the presence of murine Ig in SCID mouse serum (11). All procedures were approved by the Institute of Laboratory Animal Care and Use Committee at the Ohio State University.

Engraftment of human leukocytes into SCID mice. Human leukocytes were obtained from healthy EBV seropositive donors previously shown to generate EBV-LPD in more than $90 \%$ of engrafted hu-PBL-SCID mice. Donors were leukapheresed after obtaining written informed consent. PBMCs were isolated after Ficoll-Hypaque (Sigma Chemical Co., St. Louis, Missouri, USA) separation, enumerated and prepared for injection. For depletion of specific human lymphocyte subsets before intraperitoneal injection into SCID mice, human PBMCs were subjected to immunomagnetic bead depletion using $\mathrm{mAb}$ 's reactive against the following human antigens: CD4 (helper T cells), CD8 (cytotoxic/suppressor T cells), or CD56 and CD16 (NK cells) as described previously (8). For depletion of human monocyte/macrophages, PBMC preparations were adhered overnight to plastic culture plates at $37^{\circ} \mathrm{C}$. Efficiency of depletion was veri- fied by flow cytometric analyses and was shown to be more than $90 \%$. For depletion of endogenous murine NK cells, SCID mice were injected intraperitoneally with anti-asialo-ganglioside 1 antiserum (ASGM-1; Wako Chemicals, Richmond, Virginia, USA) as described (12, 13) 1 day before injection of human PBMC preparations and every 7 days thereafter for the duration of the study. Six-week-old SCID mice were injected intraperitoneally with $5 \times 10^{7}$ human PBMCs in $0.5 \mathrm{ml}$ of PBS. Animals injected with PBMCs depleted of specific subsets received a number of leukocytes equivalent to the percentage present in $5 \times 10^{7}$ human PBMCs. Human cell engraftment was determined by measurement of human Ig (huIg) levels in the serum of hu-PBL-SCID mice by inhibition ELISA as described previously (11).

Description of the therapentic trial. Hu-PBL-SCID mice were randomized into experimental groups to receive treatment with either PEG IL-2 (500 IU daily; Chiron Corp., Emeryville, California, USA) alone; PEG-IL-2 + ASGM-1 ( $660 \mu \mathrm{g}$ weekly); GM-CSF ( $3 \mu \mathrm{g}$ every other day; Immunex, Seattle, Washington, USA) + ASGM-1; or PEG-IL-2 + GM-CSF + ASGM-1. Cytokines were diluted in $5 \%$ dextrose water with $0.1 \%$ human albumin added as a carrier protein and were injected subcutaneously for IL-2 or intraperitoneally for GM-CSF. This trial was repeated with the PBMCs from three separate $\mathrm{EBV}^{+}$ donors, with a minimum of five animals per engraftment group. Animals in depletion experiments received cells from one of the three donors used in the initial trial, along with combined GM-CSF and PEG-IL-2 therapy.

Flow cytometric analyses. To evaluate differences in engrafted lymphocyte subsets, hu-PBL-SCID mice injected with the PBMCs from two separate donors were randomized to receive treatment with either ASGM-1 and PEG-IL-2 $(n=10)$ or ASGM-1, GM-CSF, and PEGIL-2 $(n=10)$. After treatment for 4 weeks, animals demonstrating equal engraftment (serum huIg levels) were sacrificed, and blood and organs (liver, spleen) were collected. Cell preparations from peripheral blood and spleen (dispersed into single-cell suspension) were treated with RBC lysis solution to remove murine erythrocytes, and cells were preincubated on ice with an excess of mouse Ig. Cells were next stained with directly conjugated mAb's and analyzed as described previously (14). mAb's specific for the following surface antigens were used: human CD45 (hu-CD45-FITC), huCD3 (leu4-phycoerythrin [PE] or allophycocyanin $[\mathrm{APC}]$ ), huCD4 (leu3A-PE), huCD8 (leu2A-PE), huCD19 (leu12-PE), huHLA-DR (PE), huCD86-PE, huCD11aPE, huCD1a-PE (Becton Dickinson BioSciences, San Jose, California, USA), and huCD56 (NKH1-PE; Beckman, Coulter, Miami, Florida, USA).

Human HLA B8 tetramers complexed with immunodominant peptides from the EBNA-3A latent gene (FLRGRAYGL), abbreviated FLR, and BZLF-1 lytic gene (RAKFKQLL), abbreviated RAK, and conjugated with APC were used in experiments to determine whether engrafted $T$ cell subsets were antigen-specific (15). Tetramers were provided by the NIAID 


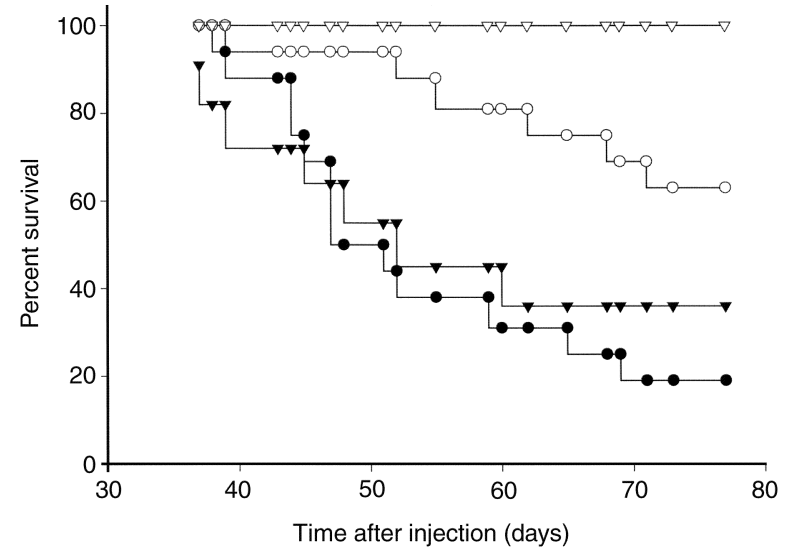

Figure 1

Combined therapy with human GM-CSF and low-dose IL-2 significantly improves survival of hu-PBL-SCID mice in the absence of murine NK cells. Survival curves are shown for hu-PBL-SCID mice treated with daily subcutaneous injections of 500 IU of PEG-IL-2 (open triangles), $3 \mu \mathrm{g}$ GM-CSF intraperitoneal injections every other day + IL-2 + ASGM-1 weekly (open circles), GM-CSF + ASGM-1 (filled triangles), or IL-2 + ASGM-1 (filled circles) after intraperitoneal injection of $50 \times 10^{6}$ human PBMCs.

Tetramer Facility and the NIH AIDS Research and Reference Reagent Program (Atlanta, Georgia, USA). Cell culture. Splenocyte preparations from Hu-PBLSCID mice treated with GM-CSF + IL-2 (100pM) + ASGM-1, or IL2 + ASGM-1 were incubated in vitro with growth medium (RPMI 1640, 10\% FBS, $1 \%$ antibiotics) supplemented with either IL-2 alone or IL-2 + GM-CSF $(1 \mu \mathrm{g} / \mathrm{ml})$. A total of $50 \%$ of medium was replaced with fresh medium every other day for up to 14 days.

Statistical analysis. The survival times of the groups were compared using the log rank test, with $P<0.05$ considered statistically significant. This test was performed with the assumption that hazard ratios are constant over time. To assess whether this assumption was met, the $\log (-\log )$ survival plots were examined. The assumption was met if the plots for each group were parallel (16).

\section{Results}

Fifty-four SCID mice were each injected with $5 \times 10^{7}$ PBMCs from three normal healthy human donors previously shown to generate EBV-LPD in more than
$90 \%$ of engrafted animals. Treatment groups included (a) IL-2 alone (500 IU/d; $n=11)$, (b) ASGM-1 (660 $\mu \mathrm{g}$ weekly) and IL-2 $(n=16)$, (c) ASGM-1 and GMCSF ( $3 \mu \mathrm{g}$ every other day; $n=11)$, and (d) ASGM-1, GM-CSF, and IL-2 $(n=16)$. Human cell engraftment was determined by serum human Ig levels and was found to be equal in all treatment groups. As shown in Figure 1, treatment with low-dose IL-2 alone prevented the development of spontaneous EBV-LPD in $100 \%$ of animals. When animals were depleted of murine NK cells by weekly injection with ASGM-1 antisera, treatment with IL-2 or GM-CSF alone failed to provide adequate protection $(19 \%$ and $36 \%$ survival, respectively), and the majority of engrafted animals developed fatal EBV-LPD, extending observations reported in earlier work $(8,17)$. Therapy with GM-CSF and IL-2 significantly improved the survival of hu-PBL-SCID mice compared with treatment with IL-2 alone $(65 \% ; P=0.0041)$. This protection was afforded in the absence of murine NK cells, suggesting cytokine cooperation with engrafted human effector cell population(s).

To determine which human mononuclear cell subset(s) was participating in the observed protective effect, we initiated an additional trial using PBMCs from a single EBV-seropositive donor. All SCID mice were pretreated with ASGM-1 antisera, received combination GM-CSF and IL-2 therapy, and were injected with whole PBMCs or PBMCs depleted of specific human mononuclear cell subsets (five animals per group), as shown in Table 1. Human Ig levels were comparable in all groups. At the end of week $12,60 \%$ of animals engrafted with whole PBMCs and treated with combination GM-CSF and IL-2 therapy survived without any evidence of EBV-LPD. In contrast, survival of hu-PBL-SCID mice engrafted with PBMCs depleted of either human $\mathrm{CD}^{+} \mathrm{T}$ cells or monocytes was reduced to $20 \%$, and animals receiving human NK cell-depleted PBMCs all died, despite each group receiving combined therapy with GM-CSF and IL-2. All deaths were attributed to lethal tumor burden. Thus, it appeared that combined cytokine therapy with GM-CSF and IL-2 required at least three separate human mononuclear cell subsets to provide significant protection against EBV-LPD in the hu-PBL-SCID mouse.

\section{Table 1}

Survival of SCID mice engrafted with whole PBMCs or PBMCs-depleted of specific human mononuclear cell subsets, and then treated with GM-CSF and IL-2

\begin{tabular}{lcccc}
\hline Injected PBMC & Method of depletion ${ }^{\mathrm{A}}$ & No. of animals & Hu-lg $^{\mathrm{B}}$ & Survival $^{\mathrm{C}}$ \\
Hu PBMC & - & 5 & $1,250 \pm 220$ & $60 \%$ \\
Hu NK depleted & Anti-CD56, Anti-CD16 & 5 & $980 \pm 120$ & $0 \%$ \\
Hu CD8 depleted & Anti-CD8 Purge & 5 & $1,120 \pm 280$ & $20 \%$ \\
Hu Monocyte & plastic adherence & 5 & $1,230 \pm 370$ & $20 \%$ \\
\hline
\end{tabular}

SCID mice were injected intraperitoneally with $50 \times 10^{6}$ human PBMCs depleted of specific human mononuclear cell subsets and were treated with daily subcutaneous injections of 500 IU PEG-IL-2, $3 \mu \mathrm{g}$ GM-CSF intraperitoneal injections every other day, and ASGM-1 intraperitoneal injections weekly. ${ }^{A}$ Subsets were depleted as indicated, or by using ex vivo immunomagnetic bead depletion. ${ }^{\text {BHuman }}$ Ig is measured in micrograms per milliliter. ${ }^{C}$ As measured at end of week 12. 


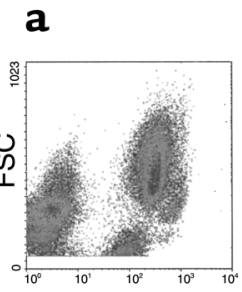

Hu-CD45

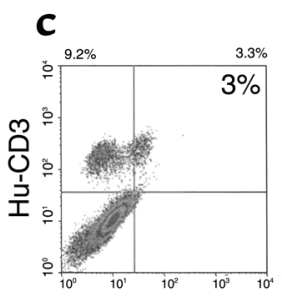

Hu-CD8

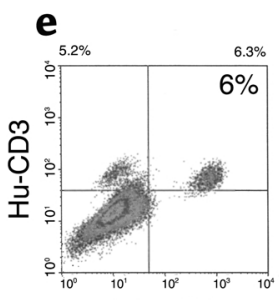

Hu-CD8

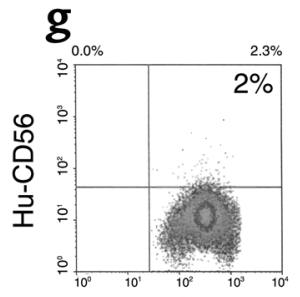

Hu-CD45

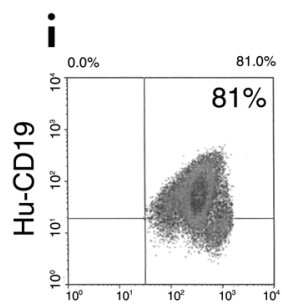

Hu-CD45

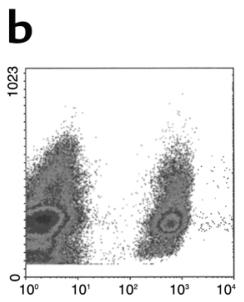

Hu-CD45
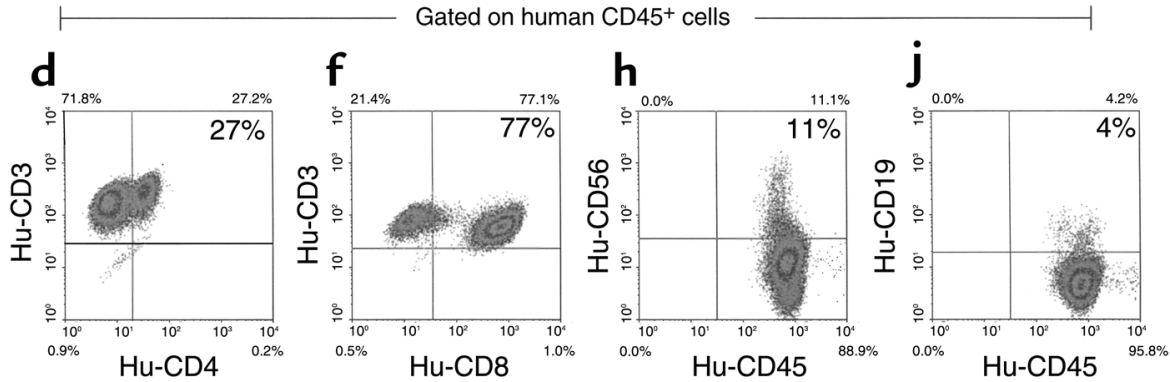

\section{Figure 2}

hu-PBL-SCID mice treated with IL-2 therapy demonstrate distinctly different populations of isolated human leukocytes in their spleens at week 4 compared with hu-PBL-SCID mice treated with GM-CSF and IL-2. Flow cytometric analysis of splenocytes from SCID mice 4 weeks after intraperitoneal injection of $50 \times 10^{6}$ human PBMCs and the initiation of treatment with either daily subcutaneous injections of 500 IU PEG-IL-2 + ASGM-1 weekly (a, c, e, g, and i) or $3 \mu \mathrm{g}$ GM-CSF intraperitoneally every other day + IL-2 + ASGM-1 (b, d, $\mathbf{f}, \mathbf{h}$, and $\mathbf{j}$ ). Each group included ten animals, with comparable human Ig levels. ( $\mathbf{a}$ and $\mathbf{b}$ ) Forward scatter (size index) versus reactivity with anti-human CD45-FITC to distinguish cells of human origin. (c-j) Human leukocyte subset analysis of this CD45 $5^{+}$population.

To investigate potential mechanisms that might account for this improved survival in vivo, two additional trials were performed with two different human donors. In the first trial, SCID mice were injected with human PBMCs and treated with ASGM-1 + IL-2, or ASGM-1 + combination therapy with GM-CSF and IL-2. Eight weeks later, engrafted hu-PBL-SCID mice from both treatment groups were sacrificed, and spleen cells were harvested. Flow cytometric analysis gating on human cells with combinations of antibodies specific for the dendritic cell phenotype failed to detect dendritic cells in either treatment group. In the second trial, SCID mice were injected with human PBMCs and treated with ASGM-1 + IL-2, or ASGM-1 + combination therapy with GM-CSF and IL-2. Four weeks later, mice from both treatment groups were sacrificed, and spleen cells were then cultured for an additional 7 days ex vivo. Mice treated with IL-2 had splenocytes cultured ex vivo in IL-2 (100 pM), whereas mice treated with IL-2 + GM-CSF had splenocytes cultured ex vivo in IL-2 $(100 \mathrm{pM})+\mathrm{GM}-\mathrm{CSF}(1 \mu \mathrm{g} / \mathrm{ml})$. A greater percentage of human cells cultured ex vivo from the spleens of mice treated with GM-CSF + IL-2 expressed the human "DC phenotype" compared with mice treated with IL-2 alone [HLA-DR ( $72 \%$ vs. $26 \%$ ), CD1a (30\% vs. 9\%), CD11a (41\% vs. $9 \%$ ), and CD86 (B7.1) (18\% vs. $6 \%$ )].

Importantly, hu-PBL-SCID mice treated with IL-2 therapy had distinctly different populations of isolated human leukocytes in their spleens at week 4 compared with hu-PBL-SCID mice treated with GM-CSF and IL-2 (Figure 2). Chimeric mice treated with IL-2 alone demonstrated three distinct populations of human leukocytes, clearly predominated by a homogeneous group of large cells with relatively intermediate CD45 surface density expression (Figure 2a). Inspection of this human population in Figure 2c, e, g, and i, shows it to be overwhelmingly composed of human $\mathrm{B}$ cells displaying a $\mathrm{CD} 45^{+} \mathrm{CD} 3^{-} \mathrm{CD} 56^{-} \mathrm{CD} 19^{+}$phenotype. B cells represent between $5 \%$ and $10 \%$ of human cells injected into SCID mice, whereas these hu-PBL-SCID mice treated with IL-2 alone have B cells that comprise more than $80 \%$ of human leukocytes. Thus, this expanded B cell population at week 4 likely represents the outgrowth of EBV-transformed lymphoblasts that is often subsequently seen in hu-PBL-SCID mice at weeks 8-12 with lethal EBV-LPD. Indeed, the vast majority of these $\mathrm{CD}^{2} 5^{+} \mathrm{CD} 19^{+}$cells coexpressed $\mathrm{CD} 23$ (data not shown), a surface marker commonly expressed on EBV transformed lymphoblasts $(18,19)$. In contrast to the huPBL-SCID mice treated with IL-2 alone (Figure 2a), those treated with GM-CSF and IL-2 have a relatively homogeneous group of smaller $\mathrm{CD}^{4} 5^{+}$cells (Figure $2 \mathrm{~b}$ ). In comparing Figure $2 \mathrm{i}$ with Figure $2 \mathrm{j}$, it is clear that huPBL-SCID mice treated with GM-CSF and IL-2 lack this distinct $B$ cell population ( $4 \%$ of total human leukocytes in Figure 2j). Inspection of figure 2, panels d, $f, h$, and $j$, reveals that the vast majority (> 96\%) of these human cells are $\mathrm{CD}^{+} \mathrm{T}$ cells. Within the $\mathrm{CD}^{+} \mathrm{T}$ cell population, the majority (> 70\%) coexpress $\mathrm{CD}^{+}$(Figure $2 \mathrm{f}$ ). Indeed, the CD4/CD8 ratio of human cells injected $(\sim 2: 1)$ compared with human cells engrafted at week 4 $(\sim 1: 3.5)$ is completely reversed in the mice treated with 
GM-CSF and IL-2 (Figure 2, $d$ and f). Although a comparable CD4/CD8 ratio is seen in hu-PBL-SCID mice treated with IL-2 alone (Figure 2, $\mathrm{c}$ and e), T cells comprise less than $15 \%$ of the total human lymphocyte population in this group.

To determine whether these human $\mathrm{CD}^{+} \mathrm{T}$ cells could be contributing to the protective effect of combination cytokine therapy with GM-CSF and IL-2, HLA tetramers complexed with immunodominant lytic (BZLF-1-derived RAK) or latent (EBNA 3A-derived FLR) EBV peptides were used to determine whether the expanded populations of human $\mathrm{CD}^{+}$subsets were
EBV-specific. Twenty SCID mice were engrafted with PBMCs from an EBV-seropositive donor (HLA A1/A1, B8/B8), treated weekly with ASGM-1 and daily with either IL-2 alone or combination therapy with GM-CSF and IL-2. Animals were sacrificed 4 weeks later, and splenocytes were collected for phenotypic analyses, gating on the human $\mathrm{CD}_{4} 5^{+}$cells. $\mathrm{CD}^{+} \mathrm{T}$ cell analysis clearly demonstrated that a fraction of the human $\mathrm{T}$ cells in the chimeric mice treated with GM-CSF and IL-2 were EBV-specific, whereas T cells from animals treated with IL-2 alone failed to demonstrate reactivity with the EBV lytic (Figure 3a) or latent (Figure 3b) pep- a
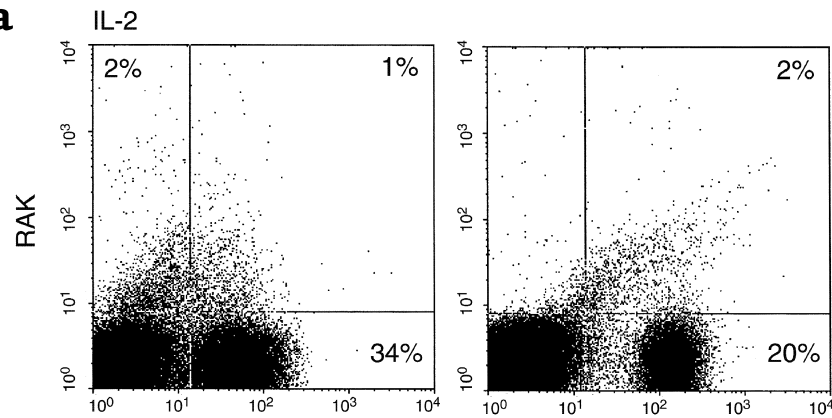

$\mathrm{IL}-2+\mathrm{GM}-\mathrm{CSF}$
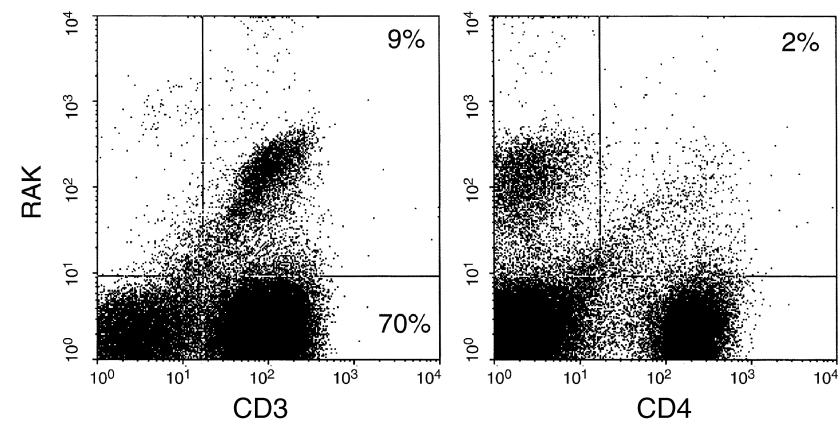

b
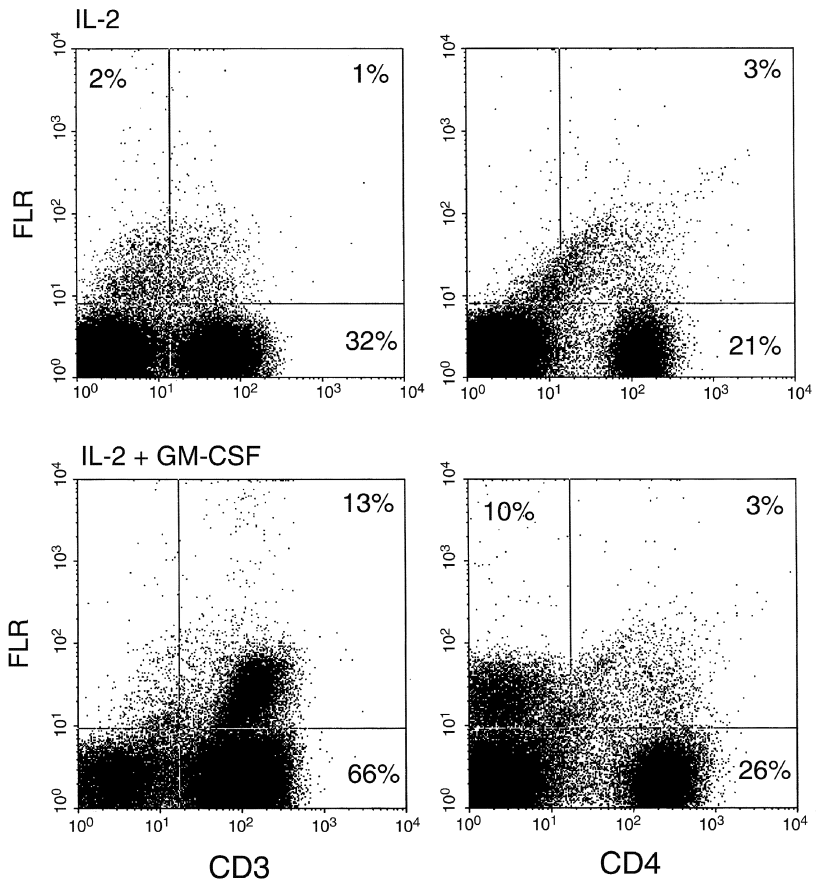
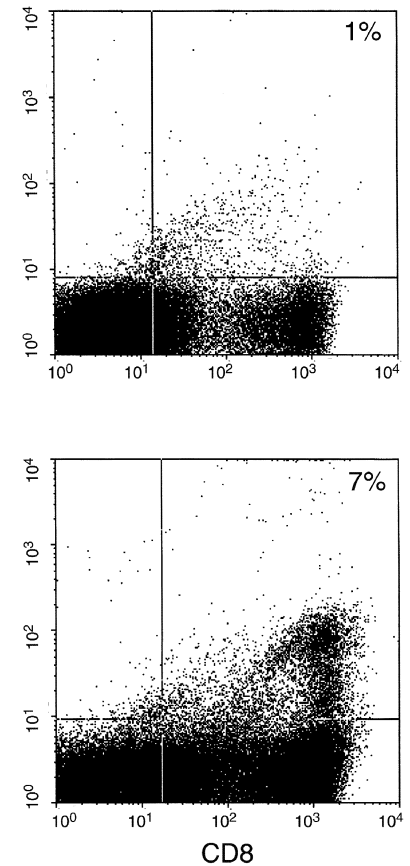

\section{Figure 3}

Combination therapy with GM-CSF and IL-2, but not IL-2 alone, induces expansion of EBV-specific T cells in vivo. Flow cytometric analysis of splenocytes from SCID mice engrafted with PBMCS from an EBV-seropositive donor (HLA A1/A1, $\mathrm{B} 8 / \mathrm{B} 8$ ), and treated weekly with ASGM1 and daily with either IL-2 alone (upper panels) or GM-CSF and IL-2 (lower panels). HLA tetramers complexed with immunodominant (a) lytic (RAK) or (b) latent (FLR) EBV peptides were used to identify expanded populations of human $\mathrm{CD}^{+}$subsets with EBV-specificity. 
tides above background staining. In the group treated with GM-CSF and IL-2, T cell specificity directed against the EBV lytic and latent epitopes could only be demonstrated in the $\mathrm{CD}^{+}$(or $\mathrm{CD}^{+} \mathrm{CD}^{-}$) subset. Approximately $10 \%$ of $\mathrm{CD}^{+}$cells ( $7 \%$ of $79 \% \mathrm{~T}$ cells) were reactive against the lytic immunodominant peptide (RAK) (Figure 3a), whereas approximately $13 \%$ of $\mathrm{CD}^{+} \mathrm{CD}^{-}$cells $(10 \%$ of $79 \% \mathrm{~T}$ cells) were reactive against the EBV latent immunodominant peptide (FLR) (Figure $3 \mathrm{~b}$ ). Thus, the ability of combination GM-CSF and IL-2 cytokine therapy to prevent EBV-LPD in a significant fraction of hu-PBL-SCID mice correlated with the clear induction of EBV-specific CTL in vivo.

\section{Discussion}

In this report, we demonstrate that combined therapy with GM-CSF and IL-2 can prevent or delay the development of EBV-LPD in a significant fraction of huPBL-SCID mice depleted of murine NK cells. Neither IL-2 nor GM-CSF alone afforded significant protection against the development of EBV-LPD. This suggests that each cytokine contributed a distinct and critical component that by itself was incomplete, but which in combination was sufficient to afford protection against malignant $B$ cell outgrowth. The selective depletion experiments showed that this protective effect of combined cytokine therapy required the presence of at least three distinct populations of human mononuclear cells. First, we demonstrated that depletion of human monocytes results in a decrease in survival ( $20 \%$ survival) due to lethal tumor burden compared with animals engrafted with whole PBMCs (60\% survival). Given that previous studies, which did not include cytokine therapy, showed poor engraftment of the monocyte/macrophage population (20), it is reasonable to infer that a potential mechanism for the protective effect seen with combined cytokine therapy may involve enhanced survival of human antigen-presenting cells in the hu-PBL-SCID chimeras. There is abundant evidence in the literature that GM-CSF not only promotes the survival of monocytes/ macrophages, but can also mediate the activation of these innate immune effector cells. This, in turn, may translate into direct anti-tumor effects, involving ADCC, Fc-dependent phagocytosis, respiratory burst, and superoxide anion generation (21-24). Additionally, activated monocytes/macrophages produce cytokines, such as IL-12, IL-15, and IL-18, which have been shown to enhance the function of other potential effector cell populations, including NK cells (25-27). GM-CSF has also been shown to improve the antigenpresenting function of monocytes/macrophages by upregulating the expression of MHC class II molecules (28), as well as several cell surface adhesion molecules (29). Fresh human cells recovered from hu-PBL-SCID mice treated for 8 weeks with IL-2 alone or GM-CSF and IL-2 failed to show any dendritic cells by flow cytometric analysis. However, a relative abundance of human cells with a dendritic cell phenotype could be found in the cultured splenocytes taken from hu-PBLSCID mice treated with GM-CSF and IL-2, compared with those collected and cultured from mice treated with IL-2 alone. This suggests that quantitative differences in small numbers of antigen presenting cells or their precursors may have existed in vivo.

It is unclear whether IL-2 has any effect upon engraftment and/or function of human antigen-presenting cells in hu-PBL-SCID mice, or whether its protective effect is mediated through lymphocyte activation alone. However, as monocytes and macrophages are known to express IL-2 receptors (30), the possibility for IL-2-induced activation of this population does exist.

Our depletion studies also showed that human NK cells are essential for the protective effect of combined cytokine therapy in the hu-PBL-SCID model. In fact, none of the mice receiving human NK-depleted PBMCs survived, compared with $60 \%$ survival in the control group. Monocyte-derived cytokines, such as IL-12, IL-15, and IL-18 enhance NK cell-mediated cytotoxicity and IFN- $\gamma$ production (25-27), the latter of which is likely critical for early effective monocyte/macrophage function (31-34), but may also have both direct and indirect anti-tumor effects (35-37). Another potential mechanism that might account for improved survival in the GM-CSF and IL-2 treated hu-PBL-SCID mice is the production of lymphokine-activated killer cells (LAKs), which are typically generated by incubating lymphoid cells with IL-2. These MHC-unrestricted cytolytic cells may develop from several different precursors, including NK cells and T cells (38). Studies in patients undergoing immunotherapy for certain types of cancer have shown that GM-CSF can augment lowdose IL-2 LAK induction from human PBMCs (39). While we have demonstrated that human NK cells are required for the prevention of EBV-LPD in this model, they clearly must function in concert with human monocytes and/or $\mathrm{CD} 8^{+} \mathrm{T}$ cells.

Finally, our depletion experiments clearly established that human $\mathrm{CD}^{+} \mathrm{T}$ cells are a critical cellular component of the protection afforded by the combination of GM-CSF and IL-2. It has been well documented that cytotoxic $\mathrm{CD}^{+} \mathrm{T}$ cells play an important role in controlling the Epstein-Barr virus during primary infection, as well as in the long-term carrier state $(40,41)$. These EBV-specific CTLs have been shown to recognize both the lytic and latent cycle EBV peptides in an MHC class I-restricted manner $(42,43)$. Here, we show that animals treated with combination GM-CSF and IL-2 demonstrate significant expansion of a $\mathrm{CD}^{+} \mathrm{CD}^{+}$subset that specifically bound HLA tetramers complexed with either a latent or lytic EBV immunodominant peptide and that similar antigen-specific $\mathrm{CD}_{3}{ }^{+} \mathrm{CD}^{+}$ lymphocytes were absent in mice treated with IL-2 alone. The likelihood that these expanded EBV-specific $\mathrm{CD}^{+} \mathrm{T}$ cells are involved in the protective effect of combined cytokine therapy in our model is supported by recent studies that showed a correlation between massive expansion of EBV-specific $\mathrm{CD}^{+} \mathrm{T}$ cells and 
clinical regression of EBV-LPD $(4,44)$, as well as a number of adoptive cellular immunotherapy studies with EBV-specific cytotoxic T cells (45-48). We provide what we believe to be the first evidence that combination cytokine therapy can promote the endogenous expansion of EBV-specific $\mathrm{CD}^{+} \mathrm{CD}^{+} \mathrm{T}$ cells. This also points to the utility of this preclinical model to study such complex interactions involving multiple human cellular subsets in vivo. Several mechanisms may be operative in promoting this expansion and may include enhancement of monocyte engraftment, followed by differentiation into a more "professional" antigen-presenting cell capable of contributing to activation of both innate and adaptive immune responses. Exogenous low-dose IL-2 would function to enhance the expansion of EBV-specific CD8 ${ }^{+} \mathrm{CTLs}$, which may provide targeted immune surveillance critical to the protective effect observed in our studies. Specific depletion of the $\mathrm{CD}^{+}{ }^{+} \mathrm{T}$-cell population was not included in these trials because prior studies conducted by our laboratory have shown that $\mathrm{CD}^{+} \mathrm{T}$ cell-mediated prevention of EBV-LPD can occur in the absence of human $\mathrm{CD} 4^{+} \mathrm{T}$ cells as long as the Th1 cytokine IL- 2 is provided exogenously (8).

It is interesting that we observed an equal expansion of $\mathrm{CD}^{+} \mathrm{CD}^{+} \mathrm{T}$ cell subsets that specifically recognized immunodominant peptides derived from latent (EBNA $3 \mathrm{~A})$ and lytic (BZLF-1) proteins. These findings point to the importance of lytic gene expression in the pathogenesis of EBV-LPD in the hu-PBL-SCID model. Recent work by Fu et al. demonstrates that activated $\mathrm{CD} 4^{+} \mathrm{T}$ cells are capable of driving BZLF-1 gene expression in resting $\mathrm{EBV}^{+}$lymphocytes and that this process is dependent upon Th2 cytokines and CD40-CD40L interaction (49). Indeed others have shown that $\mathrm{CD}^{+} \mathrm{CD}^{+} \mathrm{T}$ cells are required for EBV-associated lymphomagenesis in this model and that blockage of CD40-CD40L interaction protects animals from developing fatal EBV-LPD. Furthermore, Porcu et al. have recently shown that patients with regressing PTLD demonstrate similar expansion of $\mathrm{CD}^{+} \mathrm{CD}^{+}$lymphocytes specific for immunodominant peptides derived from the BZLF-1 protein (3). Collectively, these observations suggest that lytic gene expression may play a more prominent role in the pathogenesis of EBV-LPD than previously thought.

Given the capacity of combined GM-CSF and IL-2 cytokine therapy to promote the expansion of EBV-specific $\mathrm{T}$ cells, and to prevent or delay the development of EBV-LPD in hu-PBL-SCID mice utilizing only human effectors, it is reasonable to speculate that this approach might be useful in preventing or treating viral-associated malignancies in humans, and possibly other tumor types in which immune therapies show promise. In fact, this cytokine combination was studied in a phase IB clinical trial involving patients with incurable solid tumors. Although little change was observed in the patients' large primary tumors, half of the patients with renal cell carcinoma involving the lung had greater than 50\% reduction in their pulmonary metastases (50). This approach should be much better suited to EBV-LPD, as, unlike most solid tumors, these malignancies are easily recognized by the immune system through their expression of welldefined viral antigens $(40,43,51,52)$. Concerns relating to the survival of allografts in patients with PTLD would need to be addressed. However, in patients with $\mathrm{EBV}^{+}$tumors and $\mathrm{CD}^{+} \mathrm{T}$ cells (e.g., certain Hodgkin disease and HIV-associated non-Hodgkin lymphoma), such cytokine therapy should be considered in states of minimal residual disease. Ultimately, the concept of combination cytokine therapy could be linked with delivery of immunodominant peptides, EBV DNA, tumor cell lysates or inactivated virus to boost a patient's frequency of EBV-specific $T$ cells. This vaccine strategy could be useful for patients suffering from EBV-associated malignancies, or, preferably, as a preventive agent for those identified to be at high risk, such as patients with congenital immunodeficiency or those awaiting solid organ transplantation.

\section{Acknowledgments}

This work was supported by grants from the NIH (P30CA-16058, CA09581, CA65670, and T32 CA09338).

1. Liebowitz, D. 1998. Epstein-Barr virus and a cellular signaling pathway in lymphomas from immunosuppressed patients. N. Engl. J. Med. 338:1413-1421.

2. Lucas, K.G., Small, T.N., Heller, G., Dupont, B., and O’Reilly, R.J. 1996. The development of cellular immunity to Epstein-Barr virus after allogeneic bone marrow transplantation. Blood. 87:2594-2603.

3. Porcu, P., et al. 2001. Post-transplant lymphoproliferative disorders following renal allografting: prospective immunological and molecular monitoring identifies correlates of clinical response to reduction of immune suppression and antiviral therapy. Proc. Am. Assoc. Cancer Res. 42:S84 (Abstr.)

4. Khatri, V.P., et al. 1999. Endogenous CD8+ T cell expansion during Epstein-Barr virus-associated posttransplant lymphoproliferative disorder. J. Immunol. 163:500-506.

5. Swinnen, L.J. 2000. Diagnosis and treatment of transplant-related lymphoma. Ann. Oncol. 11:S45-S48.

6. Rowe, M., et al. 1991. Epstein-Barr virus (EBV)-associated lymphoproliferative disease in the SCID mouse model: implications for the pathogenesis of EBV-positive lymphomas in man. J. Exp. Med. 173:147-158.

7. Baiocchi, R.A., et al. 1995. Lymphomagenesis in the SCID-hu mouse involves abundant production of human interleukin-10. Blood. 85:1063-1074.

8. Baiocchi, R.A., and Caligiuri, M.A. 1994. Low-dose interleukin 2 prevents the development of Epstein-Barr virus (EBV)-associated lymphoproliferative disease in scid/scid mice reconstituted i.p. with EBV-seropositive human peripheral blood lymphocytes. Proc. Natl. Acad. Sci. USA. 91:5577-5581.

9. Baiocchi, R.A., et al. 1997. Phenotypic and functional analysis of fas (CD95) expression in primary central nervous system lymphoma of patients with Acquired Immune Deficiency Syndrome. Blood. 90:1737-1746.

10. Bosma, G.C., et al. 1988. Evidence of functional lymphocytes in some (leaky) scid mice. J. Exp. Med. 167:1016-1033.

11. Mosier, D.E., Gulizia, R.J., Baird, S.M., and Wilson, D.B. 1988. Transfer of a functional human immune system to mice with severe combined immunodeficiency. Nature. 335:256-259.

12. Kasai, M., et al. 1981. In vivo effect of anti-asialo GM1 antibody on natural killer cell activity. Nature. 291:334-335.

13. Habu, S., et al. 1981. In vivo effects of anti-asialo GM1. I. Reduction of NK activity and enhancement of transplanted tumor growth in nude mice. J. Immunol. 127:34-38.

14. Stewart, C.C., and Stewart, S.J. 1994. Cell preparation for the identification of leukocytes. Methods Cell Biol. 41:39-60.

15. Burrows, S.R., et al. 2000. Peptide-MHC class I tetrameric complexes display exquisite ligand specificity. J. Immunol. 165:6229-6234.

16. Klein, J.P., and Moeschberger, M.L. 1997. Survival analysis: techniques for censored and truncated data. Springer-Verlag. New York, New York, USA. 27-30.

17. Veronese, M.L., et al. 1992. Lymphoproliferative disease in human 
peripheral blood mononuclear cell-injected SCID mice. I. T lymphocyte requirement for B cell tumor generation. J. Exp. Med. 176:1763-1767.

18. Ling, N.R., et al. 1989. A phenotypic study of cells from Burkitt lymphoma and EBV-B-lymphoblastoid lines and their relationship to cells in normal lymphoid tissues. Int. J. Cancer. 43:112-118.

19. Wendel-Hanson, V., Tao, W., Ericson, M., Klein, G., and Rosen, A. 1992 Cell phenotype (CD23)-dependent variation in EBV genome copy numbers within lymphoblastoid cell lines (LCL). Int. J. Cancer. 50:589-592.

20. Tary-Lehmann, M., Saxon, A., and Lehmann, P.V. 1995. The human immune system in hu-PBL-SCID mice. Immunol. Today. 16:529-533.

21. Wiltschke, C., Krainer, M., Wagner, A., Linkesch, W., and Zielinski, C.C. 1995. Influence of in vivo administration of GM-CSF and G-CSF on monocyte cytotoxicity. Exp. Hematol. 23:402-406.

22. Wing, E.J., Magee, M., Whiteside, T.L., Kaplan, S.S., and Shadduck, R.K. 1989. Recombinant human granulocyte/macrophage colony-stimulating factor enhances monocyte cytotoxicity and secretion of tumor necrosis factor-alpha and interferon in cancer patients. Blood. 73:643-646.

23. Coleman, D.L., Chodakewitz, J.A., Bartiss, A.H., and Mellors, J.W. 1988. Granulocyte-macrophage colony-stimulating factor enhances selective effector functions of tissue-derived macrophages. Blood. 72:573-578

24. Perkins, R.C., Vadjan-Raj, S., Scheule, R.K., Hamilton, R., and Holian, A. 1993. Effects of continuous high dose rhGM-CSF on human monocyte activity. Am. J. Hematol. 43:279-285.

25. Carson, W.E., et al. 1995. Endogenous production of interleukin 15 by activated human monocytes is critical for optimal production of interferon-gamma by natural killer cells in vitro. J. Clin. Invest. 96:2578-2582.

26. Carson, W.E., et al. 1994. Interleukin (IL) 15 is a novel cytokine that activates human natural killer cells via components of the IL-2 receptor. J. Exp. Med. 180:1395-1403.

27. Fehniger, T.A., et al. 1999. Differential cytokine and chemokine gene expression by human NK cells following activation with IL-18 or IL-15 in combination with IL-12: implications for the innate immune response. J. Immunol. 162:4511-4520.

28. Fischer, H.G., Frosch, S., Reske, K., and Reske-Kunz, A.B. 1988. Granulocyte-macrophage colony-stimulating factor activates macrophages derived from bone marrow cultures to synthesis of MHC class II molecules and to augmented antigen presentation function. J. Immunol. 141:3882-3888.

29. Williams, M.A., Kelsey, S.M., Collins, P.W., Gutteridge, C.N., and Newland, A.C. 1995. Administration of rHuGM-CSF activates monocyte reactive oxygen species secretion and adhesion molecule expression in vivo in patients following high-dose chemotherapy. Br. J. Haematol. 90:31-40.

30. Epling-Burnette, P.K., Wei, S., Blanchard, D.K., Spranzi, E., and Djeu, J.Y. 1993. Coinduction of granulocyte-macrophage colony-stimulating factor release and lymphokine-activated killer cell susceptibility in monocytes by interleukin-2 via interleukin-2 receptor beta. Blood. 81:3130-3137.

31. Webb, D.S., and Gerrard, T.L. 1990. IFN-alpha and IFN-gamma can affect both monocytes and tumor cells to modulate monocyte-mediated cytotoxicity. J. Immunol. 144:3643-3648.

32. McCarthy, J.B., Vachhani, B.V., Wahl, S.M., Finbloom, D.S., and Feldman, G.M. 1997. Human monocyte binding to fibronectin enhances IFN-gamma-induced early signaling events. J. Immunol. 159:2424-2430.

33. Kildahl-Andersen, O., Espevik, T., and Nissen-Meyer, J. 1985. IFNgamma-induced production of monocyte cytotoxic factor. Cell Immunol. 95:392-406.

34. Munn, D.H., and Armstrong, E. 1993. Cytokine regulation of human monocyte differentiation in vitro: the tumor-cytotoxic phenotype induced by macrophage colony-stimulating factor is developmentally regulated by gamma-interferon. Cancer Res. 53:2603-2613.

35. Ogawa, M., et al. 1998. Multiple roles of interferon-g in the mediation of interleukin 12-induced tumor regression. Cancer Res. 58:2426-2432.

36. Evans, R., Fuller, J.A., Christianson, G., Krupke, D.M., and Troutt, A.B. 1997. IL-15 mediates anti-tumor effects after cyclophosphamide injection of tumor-bearing mice and enhances adoptive immunotherapy: the potential role of NK cell subpopulations. Cell. Immunol. 179:66-73.

37. Osaki, T., et al. 1999. Potent antitumor effects mediated by local expression of the mature form of the interferon-gamma inducing factor, interleukin-18 (IL-18). Gene Ther. 6:808-815.

38. Grimm, E.A., Mazumber, A., Zhang, H.Z., and Rosenberg, S.A. 1982. Lymphokine-activated killer cell phenomenon: lysis of natural killerresistant fresh solid tumor cells by interleukin 2-activated autologous human peripheral blood lymphocytes. J. Exp. Med. 155:1823-1841.

39. Baxevanis, C.N., et al. 1995. Enhanced human lymphokine-activated killer cell function after brief exposure to granulocyte-macrophagecolony stimulating factor. Cancer. 76:1253-1260.

40. Rickinson, A.B., and Moss, D.J. 1997. Human cytotoxic T lymphocyte responses to Epstein-Barr virus infection. Ann. Rev. Immunol. 15:405-431.

41. Steven, N., Leese, A.M., Annels, N.E., Lee, S.P., and Rickinson, A.B. 1996. Epitope focusing in the primary cytotoxic $\mathrm{T}$ cell response to EpsteinBarr virus and its relationship to $\mathrm{T}$ cell memory. J. Exp. Med. 184:1801-1813.

42. Steven, N.M., et al. 1997. Immediate early and early lytic cycle proteins are frequent targets of the Epstein-Barr virus-induced cytotoxic T cell response. J. Exp. Med. 185:1605-1617.

43. Murray, R.J., et al. 1992. Identification of target antigens for the human cytotoxic T cell response to Epstein-Barr virus (EBV): implications for the immune control of EBV-positive malignancies. J. Exp. Med. 176:157-168.

44. Kuzushima, K., et al. 2000. Longitudinal dynamics of Epstein-Barr virusspecific cytotoxic $\mathrm{T}$ lymphocytes during posttransplant lymphoproliferative disorder. J. Infect. Dis. 182:937-940.

45. Papadopoulos, E.B., et al. 1994. Infusions of donor leukocytes to treat Epstein-Barr virus-associated lymphoproliferative disorders after allogeneic bone marrow transplantation. N. Engl. J. Med. 330:1185-1191.

46. Rooney, C.M., et al. 1995. Use of gene-modified virus-specific T lymphocytes to control Epstein-Barr-virus-related lymphoproliferation. Lancet. 345:9-13.

47. Rooney, C.M., et al. 1998. Infusion of cytotoxic T cells for the prevention and treatment of Epstein-Barr virus-induced lymphoma in allogeneic transplant recipients. Blood. 92:1549-1555.

48. Rooney, C.M., et al. 1998. Treatment of relapsed Hodgkin's disease using EBV-specific cytotoxic T cells. Ann. Oncol. 9:S129-S132.

49. Fu, Z., and Cannon, M.J. 2000. Functional analysis of the CD4+ T-cell response to Epstein Barr virus: T-cell-mediated activation of resting B cells and induction of viral BZLF1 expression. J. Virology. 74:6675-6679.

50. Schiller, J.H., et al. 1996. Clinical and immunological effects of granulocyte-macrophage colony-stimulating factor coadministered with interleukin 2: a phase IB study. Clin. Cancer Res. 2:319-330.

51. Murray, R.J., et al. 1990. Human cytotoxic T cell responses against Epstein-Barr virus nuclear antigens demonstrated using recombinant vaccinia viruses. Proc. Natl. Acad. Sci. USA. 87:2906-2910.

52. Khanna, R., et al. 1992. Localization of Epstein-Barr virus cytotoxic T cell epitopes using recombinant vaccina: implications for vaccine development. J. Exp. Med. 176:169-176. 